\title{
Composite Proton Electrolyte Membranes Sulfonated Poly Ether-Ether Ketone (SPEEK) at Various Amount of Mesoporous Phosphotungstic Acid (mPTA) for Hydrogen Fuel Cell Application
}

\author{
Shaymala Mehanathan ${ }^{\mathrm{a}, \mathrm{b}}$, Hilmi Mohamed ${ }^{\mathrm{a}, \mathrm{b}}$, Juhana Jaafar ${ }^{\mathrm{a} *}$, Hamid Ilbeygi ${ }^{\mathrm{c}}$ \\ aAdvanced Membrane Technology Research Centre (AMTEC), Universiti \\ Teknologi Malaysia, 81310 UTM Johor Bahru, Johor, Malaysia

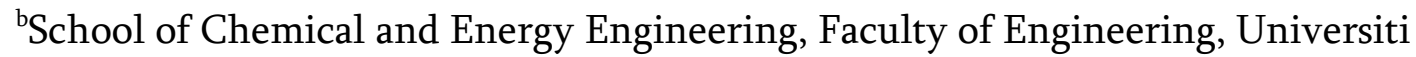 \\ Teknologi Malaysia, 81310 UTM Johor Bahru, Johor, Malaysia \\ ${ }^{\mathrm{c}}$ Future Industries Institute, University of South Australia
}

Submitted: 22/06/2020. Revised edition: 25/10/2020. Accepted: 26/10/2020. Available online: 19/11/2020

\begin{abstract}
Polymer electrolyte membrane fuel cells (PEMFC), also known as proton exchange membrane fuel cells utilise polymer electrolyte membranes (PEMs) to conduct protons for ion-exchange purposes. Nafion is a common polymeric material to be used as PEM for fuel cells. However, Nafion has major weaknesses such as low proton conductivity under anhydrous conditions at elevated temperatures due to water evaporation and continuous need for water management, low tolerance for fuel impurities and high cost. This study fabricates a newly composite PEM by blending SPEEK and mesoporous phosphotungstic acid (mPTA) at various loading in order to obtain the most favourable PEMFC power output. SP/2.0 mPTA membrane has shown outstanding properties in terms of dense structure, $35.75 \%$ of water uptake and expected an elemental mapping of $76.20 \%$ Tungsten and 23.80\% Phosphorus. Even with a lower proton conductivity of 3.502 $\mathrm{mScm}{ }^{-1}$, this membrane has a higher power density of $154.4 \mathrm{~mW} / \mathrm{cm}^{2}$ compared to the other four membranes. Owing to the unique characteristics of the as-synthesized mPTA such as high surface area, porous structure, good thermal and chemical resistance, SPEEKmPTA membrane is one of the promising materials for PEMFC applications.
\end{abstract}

Keywords. PEMFC, hydrogen fuel cell, polymer electrolyte membrane (PEM), mesoporous phosphotungstic acid (mPTA), composite PEM, inorganic fillers

\subsection{INTRODUCTION}

Hydrogen fuel cell or polymer electrolyte membrane fuel cell (PEMFC) uses an acidic polymer membrane as the electrolyte and platinum-catalysed electrodes [1]. As the name implies, the heart of the hydrogen fuel cell is the Proton Exchange Membrane (PEM).

The commercially available PEM is a perfluorinated ion-exchange 
membrane which is the Nafion. Nafion is a copolymer which is composed of perfluoroether side chains terminated with sulfonic acid groups that are randomly distributed along the semicrystalline polymer backbone of (perfluoroethylene) [2]. This class of polymer is generally utilized as a reference for PEM in polymer electrolyte fuel cell studies. The morphology of the Nafion membrane has significant impacts on its properties. The acidic region of Nafion is contributing to high proton conductivity. Moreover, Nafion possesses high-performance when operating at a temperature of $90^{\circ} \mathrm{C}$ [24].

However, it needs to be highlighted that the proton conductivity of the Nafion membrane heavily relies upon hydration conditions. In this case, water acts as the medium to promote proton conduction or transport via free diffusion and/or proton hopping (Grotthus mechanism). Unfortunately, the proton conductivity of the Nafion membrane has a high tendency to deteriorate, especially when the temperature is beyond $90^{\circ} \mathrm{C}$ due to the dehydration effect. Because of this, Nafion needs to be hydrated at a sufficient level all the time upon fuel cell operation. Thus, alternative PEMs with high water retention capability to maintain high proton conductivity as well as high thermal and mechanical stability are critically desirable [5].

The non-fluorinated polymeric membranes have advantages compared to perfluorinated membranes, owing to readily availability, processability, and good stability in fuel cell environments [6]. They can be easily designed according to the favorable properties for fuel cell applications. Typical examples of hydrocarbon polymers used as PEMs are sulfonated poly ether-ether ketone, polyarylene, polysulfone and polyimides [7-9]. They are cost-effective than fluorinecontaining based polymers.

Hybrid or composite polymerinorganic PEMs have gained so much attention due to their high chemical and mechanical stability and high proton conductivity. A variety of fillers such as zeolites and mesoporous silica materials have been used in the preparation of hybrid or composite membranes [10]. These membranes have exhibited improved performances compared to the neat polymer membranes. Heteropoly acids (HPAs) are a type of mesoporous silica material which has high acidity and proton conductivity. HPAs show high solubility in polar solvents. Their strong acidity is accounted for the large size of the polyanion that yields low delocalized charge density. Hydrated HPAs are normally embedded in a hydrophilic polymer matrix owing to their high proton conductivity and ability to improve the overall mechanical properties of the resulting polymeric membrane [11]. However, their low specific surface area and high solubility in polar solvents make them unattractive to be used in catalytic or energy applications. This obstacle can be overcome by creating nanoporous within the HPA. Therefore, in this study, modified 
heteropoly acid (mPTA), one of a HPA materials is employed. The mPTA with a spherical morphology was synthesized through the selfassembly of phosphotungstic acid (PTA) with a polymeric surfactant that was stabilized by $\mathrm{KCl}$ via hydrothermal treatment [11].

PEM comprised of mPTA incorporated sulfonated PEEK was prepared. In this study, the membranes with different mPTA loadings were characterized in terms of morphological structure, water uptake and swelling, and proton conductivity. The power output of the membranes was also evaluated through the voltage versus current density polarization characterization profile in order to determine their overall performance and desirability for the PEMFC application.

\subsection{METHODS}

\subsection{Materials}

Dimethyl sulfoxide (DMSO) with purity (GC) $\geq 99.9 \%$ was provided by Merck Co., Germany, poly-etherether ketone (PEEK) was obtained from Victrex, modified phosphotungstic acid (mPTA) was synthesized and supplied by this project collaboration team member from the University of South Australia, and sulphuric acid $\left(\mathrm{H}_{2} \mathrm{SO}_{4}\right)$ at $\quad 95-97 \%$ concentration was supplied by Merck Co, Germany.

\subsubsection{Sulfonation Process of Poly Ether Ether Ketone (PEEK)}

The process started by weighing $50 \mathrm{~g}$ of PEEK. After that, the PEEK polymer was dried in an oven at $60^{\circ} \mathrm{C}$ to remove the moisture content. Then, 1 litre of the concentrated sulphuric acid was poured into a round bottom flask equipped with a heating mantle. Simultaneously, PEEK polymer was added gradually to into the flask. The mixture was then undergone the sulfonation process via stirring with the propeller. The solution was stirred for 1 hour at room temperature. Then, the solution was continuously stirred at $50^{\circ} \mathrm{C}$ for another 3 hours. The as-sulfonated polymer was then collected by precipitating the acid polymer solution into the ice bath. The SPEEK polymer was then filtered and washed with deionized water until the $\mathrm{pH}$ became 7 . The resulting SPEEK polymer was dried in an oven.

\subsubsection{Preparation of SPEEK-mPTA Membrane}

$10 \mathrm{~g}$ of as-prepared SPEEK polymer was weighed and dissolved in $90 \mathrm{~g}$ of DMSO. The amount of mPTA was varied from 0.5 to $2.5 \mathrm{wt}$. \%. The solution was stirred overnight to ensure homogeneity. Then, the solution was cast in a petri dish and dried in an oven at $80^{\circ} \mathrm{C}$ for 24 hours then another $100^{\circ} \mathrm{C}$ for 6 hours. After that, the sample was collected. Prior to the characterization, the sample was undergone pre-treatment with 1 M sulphuric acid for 24 hours. Finally, the sample was dried in an oven. Table 1 shows the composition of the SPEEK-mPTA designated in this study. The reference for the values of SPEEK and mPTA was described elsewhere [12-14]. 
Table 1 Dope solution formulation of SPEEK-mPTA

\begin{tabular}{|c|c|c|c|}
\hline & $\begin{array}{l}\text { SPEEK } \\
\text { (wt. \%) }\end{array}$ & $\begin{array}{l}\text { DMSO } \\
\text { (wt. \%) }\end{array}$ & $\begin{array}{l}\text { mPTA } \\
\text { (wt. \%) }\end{array}$ \\
\hline SPEEK & \multirow{6}{*}{10} & \multirow{6}{*}{90} & - \\
\hline $\mathrm{SP} / 0.5 \mathrm{mPTA}$ & & & 0.5 \\
\hline $\mathrm{SP} / 1.0 \mathrm{mPTA}$ & & & 1.0 \\
\hline $\mathrm{SP} / 1.5 \mathrm{mPTA}$ & & & 1.5 \\
\hline $\mathrm{SP} / 2.0 \mathrm{mPTA}$ & & & 2.0 \\
\hline $\mathrm{SP} / 2.5 \mathrm{mPTA}$ & & & 2.5 \\
\hline
\end{tabular}

\subsection{Characterisation Method}

\subsubsection{Morphology Analysis}

Scanning Electron Microscopy (SEM) was used to observe the surface morphological structure of SPEEKmPTA membranes and dispersion of mPTA in the membranes. For sample preparation, all membranes were immersed in liquid nitrogen for a few seconds. Then, sample were broke, adhered to stub and undergone a gold coating process. The sample morphology was determined by SEM. The magnification of the sample testing was varied from $500-10000 x$. EDX mapping was carried out to observe the dispersion of mPTA in the SPEEK matrix.

\subsubsection{Water uptake and Swelling}

A water uptake test was conducted to determine the membrane capabilities in holding the water content. The membrane was dried in an oven to remove additional moisture present in the membrane. After that, the membrane was weighted. Then, the membrane was immersed in water for 24 hours and re-weighted. The formula for water uptake calculation is shown in Equation 3.1.

Water uptake $=(\mathrm{Gw}-\mathrm{Gd}) / \mathrm{Gd} \times 100 \%$ Eq. 3.1

where Gw is the weight of wet membranes and $\mathrm{Gd}$ is the weight for dry membranes.

Meanwhile, the swelling ratio was calculated to determine the dimensional stability of membranes in the water environment. The swelling ratio of PEM was determined according to the following equation:

Swelling ratio $=(\mathrm{Lw}-\mathrm{Ld}) / \mathrm{Ld} \times 100 \%$

Eq. 3.2

where Lw is the length of wet membranes and Ld is the weight of dry membranes.

\subsubsection{Proton Conductivity}

Transverse proton conductivities of membranes was measured by impedance spectroscopy over a frequency range of 1 to $107 \mathrm{~Hz}$ with 50 to $500 \mathrm{mV}$ oscillating voltage, model Solartron 1260 Gain phase Analyzer, AMETEK, Inc., UK. The hydrated membrane was placed between two stainless steel electrodes to measure the proton conductivity range from room temperature to $120^{\circ} \mathrm{C}$. The conductivity $(\sigma)$ of 
samples in the transverse direction was calculated from the impedance data using the relationship:

$\sigma=\mathrm{d} / \mathrm{RS}$

where $\mathrm{d}$ and $\mathrm{S}$ are the thickness and surface area of the membrane sample, respectively, and $\mathrm{R}$ was derived from the low intersection of the highfrequency semi-circle on a complex impedance plane with the $\operatorname{Re}(\mathrm{Z})$ axis.

\subsubsection{I-V Polarization Study}

Single PEMFC performance was evaluated by recording the cell voltage vs. current density curves using a fuel cell test system (850e) equipped with hydrogen and oxygen lines. Before the sample underwent testing, the membrane was cut in a square for about $3 \times 3 \mathrm{~cm}$. Meanwhile, anode and cathode were also cut into a square with a size of $1.5 \times 1.5 \mathrm{~cm}$. The membrane was sandwiched between the electrode and being hot pressed for 1 minute at $50 \mathrm{oC}$. The complete cell was placed in the membrane electrode assembly and all the gas tubes were connected. The condition was set to fully hydrated and the temperature was maintained at $60^{\circ} \mathrm{C}$. Then, the same procedure was repeated for other samples.

\subsection{RESULTS AND DISCUSSION}

\subsection{Surface Morphology of SPEEK- mPTA Membranes}

It is important to note that the membrane for PEMFC should be in dense membrane structure. This is because the transport of reactants such as protons and hydrogen through the electrolyte membrane is mediated by free solution diffusion and Grotthus mechanisms which relied on water transport. The highly transported protons are favorable but not for hydrogen because it will lead to poor membrane performance. Therefore, a dense membrane is desirable to manage the water, protons and hydrogen transport [15]. Figure 1 shows the morphological structure of SPEEK and SPEEKmPTA membranes. Figure 1(a) clearly shows that the parent SPEEK membrane is dense in structure. The dense structure started forming pores with 0.5 wt.\% mPTA loading. Unfortunately, further increase of mPTA loading from 1.0 wt.\% to 2.5 wt. \% tend to act as a pore former and produce porous SPEEK membrane structure. The surface SEM images revealed that the pore size of the membranes was in a dry state, which could stand for the real pore size of the mPTA membranes. The number of pores was increasing with the addition of fillers. This phenomenon occurred might be due to the rapid aggregation of mPTA fillers when the dope solution was exposed to air during the drying process. A similar observation was reported by Yang et al. (2007) as well regarding the UF membrane containing $\mathrm{TiO} 2$ fillers that tend to aggregate because of an instantaneous demixing process of the casting solution [16]. Higher mPTA loading has led to the formation of poorer and looser surface (Figure 1 (e) and 
(f)) and cross-section (Figure 2 (a) and (b)) structure as compared to the parent SPEEK membrane [17]. The loosen structure caused the mPTA concentrated at the bottom part of the membrane layer. This will consequently reduce the effective surface area of the mPTA on the membrane top surface in activating the proton conduction ability.
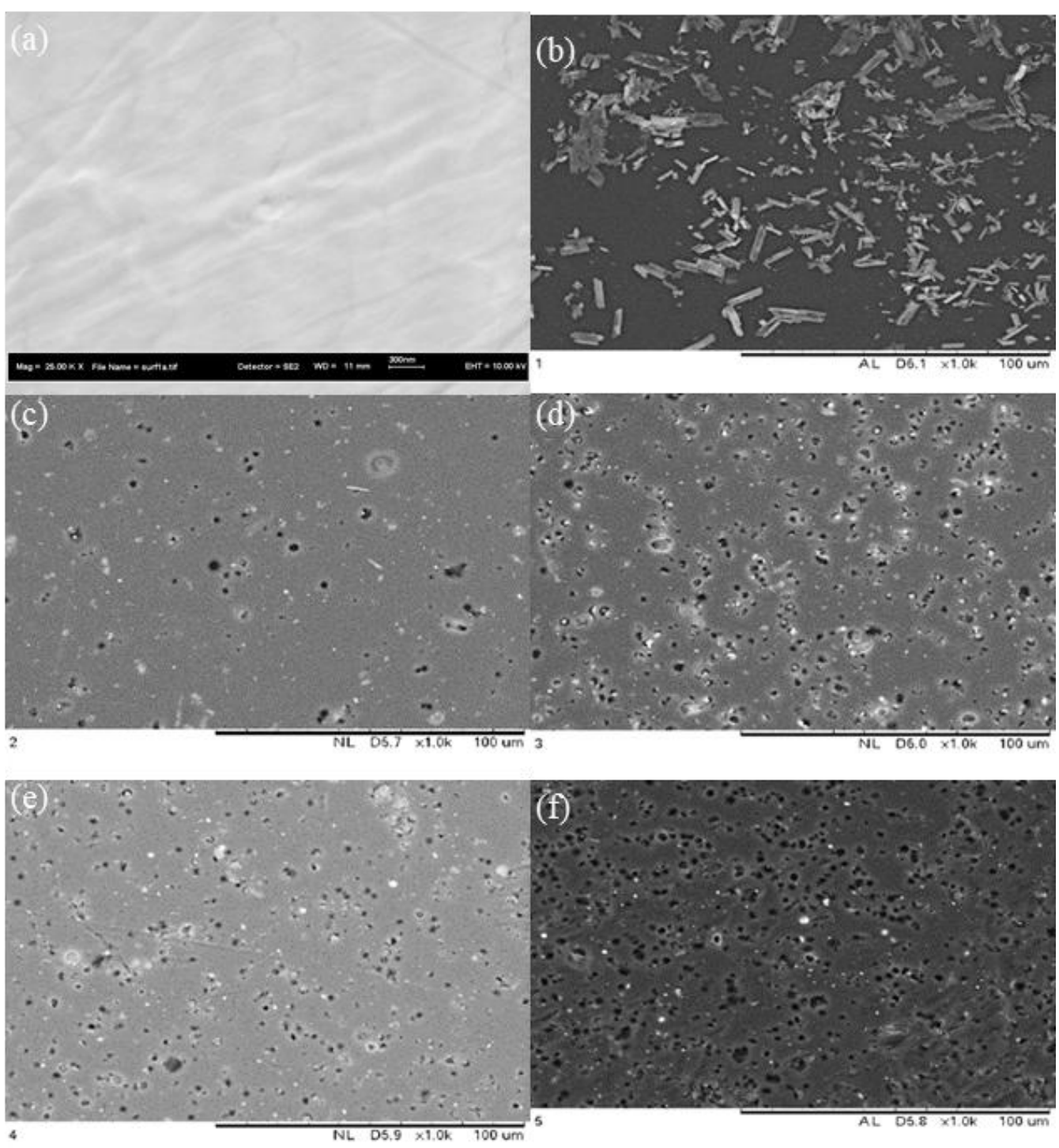

Figure 1 SEM micrographs of SPEEK (a) SPEEK with mPTA SP/0.5mPTA (b) SP/1.0mPTA (c) SP/1.5mPTA (d) SP/2.0mPTA (e) and SP/2.5mPTA (f) 

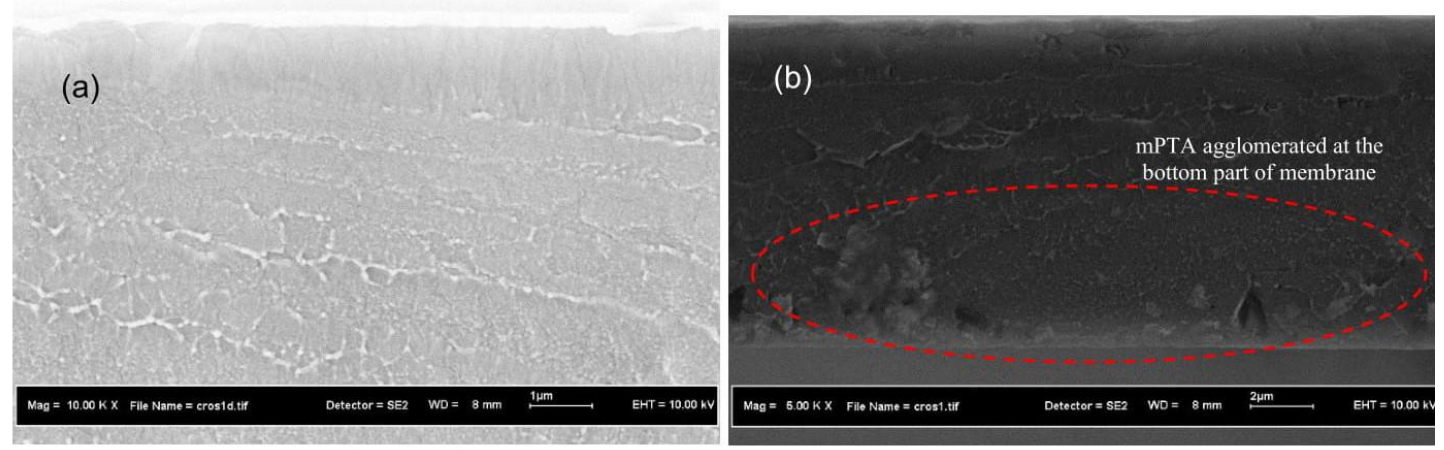

Figure 2 Cross-section images of (a) parent SPEEK membrane and (b) SP/2.5mPTA membrane

\subsection{Elemental Mapping of SPEEK- mPTA Membranes}

In order to confirm the success of integrating the $\mathrm{mPTA}$ in the SPEEK membranes, the elemental composition of phosphorus and tungsten in the SPEEK/mPTA composite membranes were analysed. Figure 3 shows the result of the elemental composition of phosphorus and tungsten in SPEEK/mPTA composite membranes. Table 2 illustrates the weight percentage of Phosphorus and Tungsten in the SPEEK-mPTA membranes. As being show in the table, an increase in mPTA content increased the weight percentage of tungsten in the membrane which is in agreement with the SEM images. When the loading of $\mathrm{mPTA}$ is at $0.5 \mathrm{wt} \%$, the weight percentage of tungsten is at $53.45 \%$ and it constantly increases with the increase of mPTA loading. Although it was claimed that tungsten can help to stabilise mPTA in the polymer matrix, there exists an optimum ratio between tungsten and phosphorus [18]. The results showed that mPTA fillers have been successfully anchored to the SPEEK membrane matrix. However, excessive MPTA loading tends to cause agglomeration of tungsten and phosphorus that will consequently reduce the effective area of mPTA towards conducting protons [18].

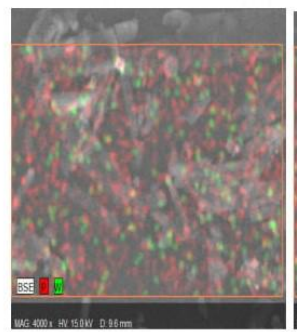

(a)

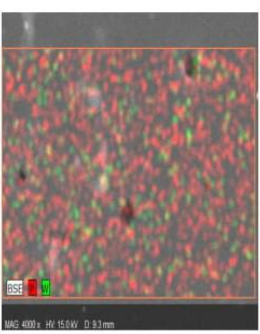

(b)

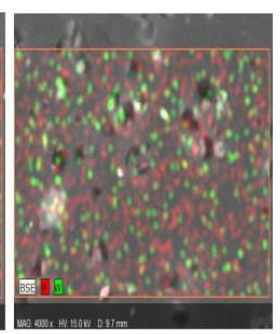

(c)

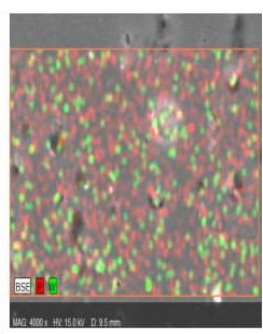

(d)

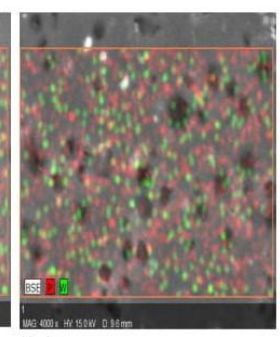

(e)

Figure 3 Elemental composition of phosphorus (red) and tungsten (green) of (a) $\mathrm{SP} / 0.5 \mathrm{mPTA}$ (b) SP/1.0mPTA (c) SP/1.5mPTA (d) SP/2.0mPTA and (e) SP/2.5mPTA composite membranes 
Table 2 Weight percentage of Tungsten and Phosphorus in membranes

\begin{tabular}{|l|l|l|}
\hline Membranes & Tungsten (wt\%) & Phosphorus (wt\%) \\
\hline SP/0.5mPTA & 53.45 & 46.55 \\
\hline SP/1.0mPTA & 60.62 & 39.38 \\
\hline SP/1.5mPTA & 67.42 & 32.58 \\
\hline SP/2.0mPTA & 76.20 & 23.80 \\
\hline SP/2.5mPTA & 86.63 & 13.37 \\
\hline
\end{tabular}

3.3 Water Uptake and Swelling Behavior of SPEEK/mPTA Membranes

Water uptake reflects the capability of a membrane to absorb and hold water that is desirable for proton conductivity. Water molecules can help vehicle and Grotthuss mechanisms by providing proton carriers and forming hydrogen bond networks, respectively [19]. Water uptake of SPEEK membranes with different mPTA loadings at room temperature is presented in Figure 4. The results showed that the SPEEK/mPTA composite membranes generally exhibit higher water uptake with an increase in mPTA loading which suggested that the volume of water channels was also increased. This was due to the strong polar interaction between water molecules and the acid sites on the surface of the mPTA [20]. The interaction mechanism between the water molecules and the MPTA is illustrated in Figure 5. It was also found that the water uptake tends to decrease beyond 2.0 wt.\% of mPTA loading. This phenomenon can be correlated to the morphological structure of the SPEEK/mPTA that seems to exhibit severe agglomeration due to an increase of the mPTA loading. This will consequently decrease the effective surface area of the mPTA particles, leading to the decrease of the water uptake.

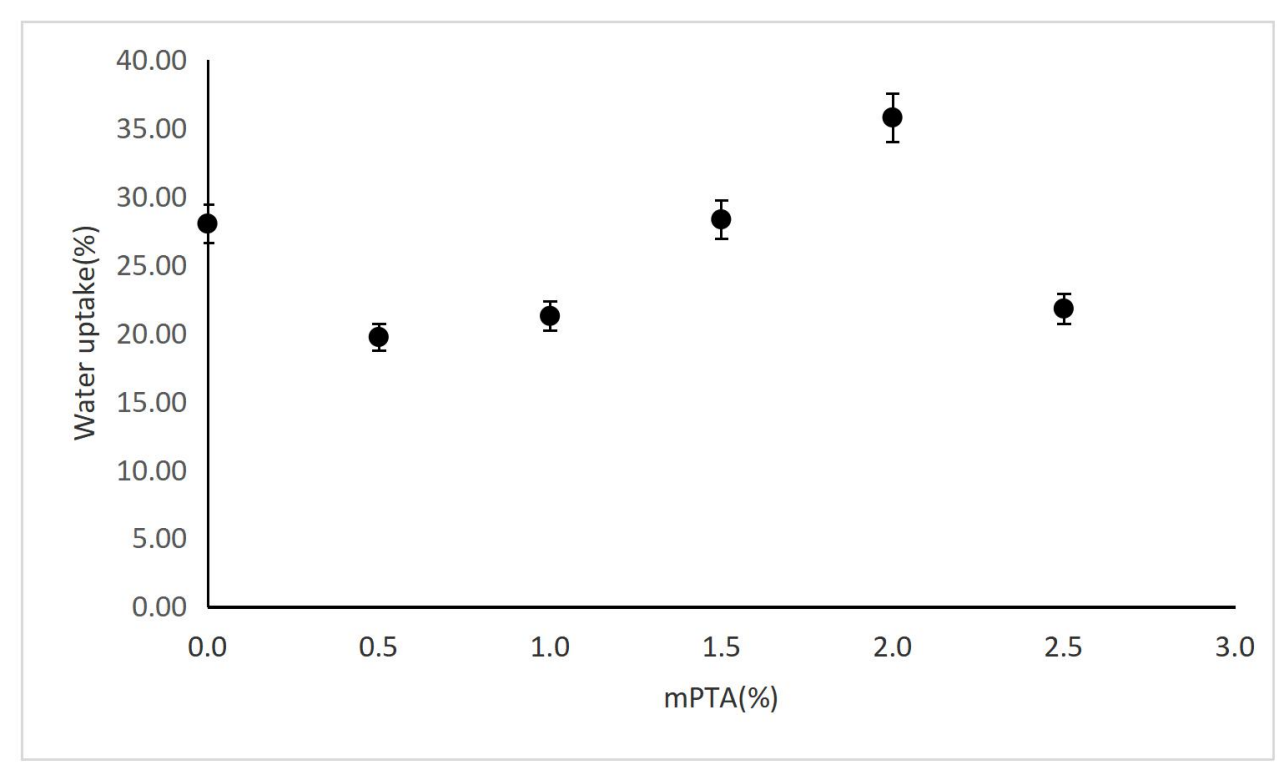

Figure 4 Percentage of water uptake of membranes at different mPTA loading 


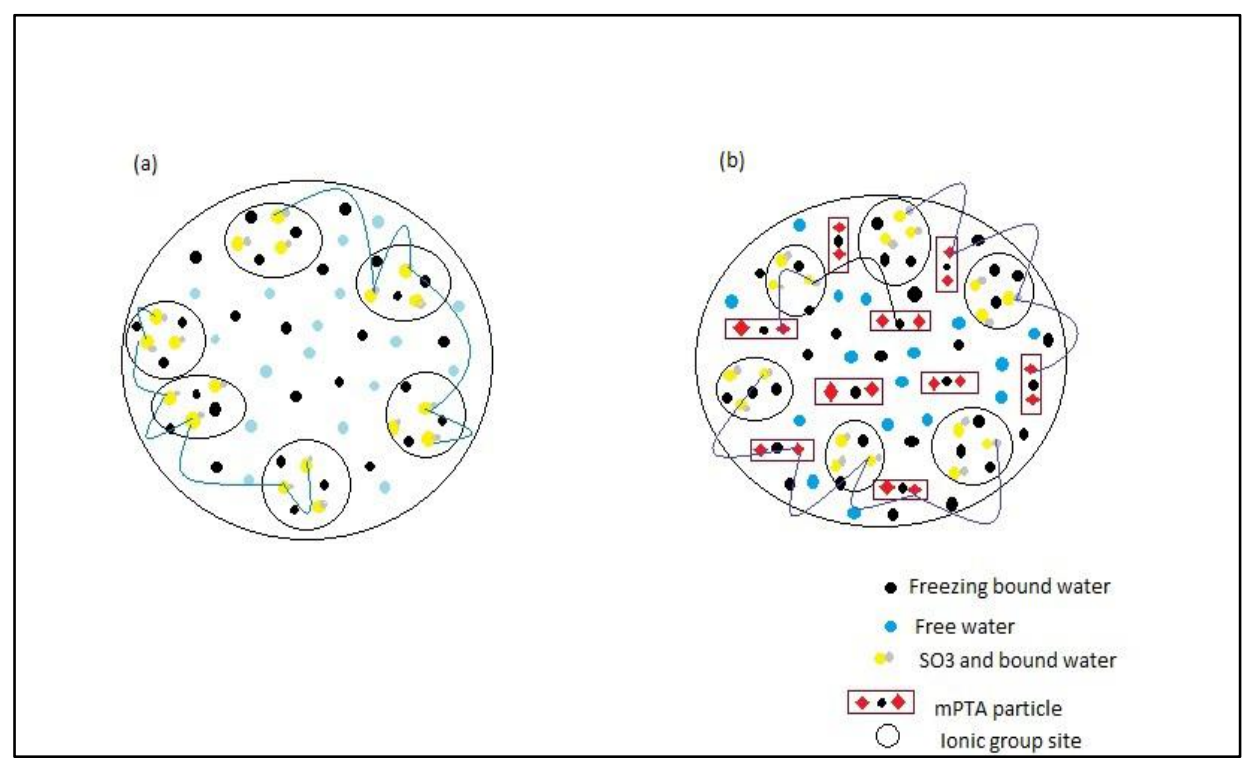

Figure 5 Illustration about plausible transportation of proton in (a) parent SPEEK (b) $\mathrm{SP} / \mathrm{mPTA}$ membrane

The high swelling in membranes causes low durability, low mechanical stability and eventually a low fuel cell performance [21]. From Figure 6, the swelling ratio results of the resulting SPEEK/mPTA membranes fluctuate. However, when the SPEEK composite membranes are compared to parent SPEEK, it is worth mentioning that the swelling ratio decreased upon the addition of mPTA loading. The interaction between MPTA and SPEEK matrix through hydrogen bonding between acid groups of mPTA and sulfonic acid groups of SPEEK restricted the movement of SPEEK polymer chains and consequently contributed to better dimensional stability composite membranes.

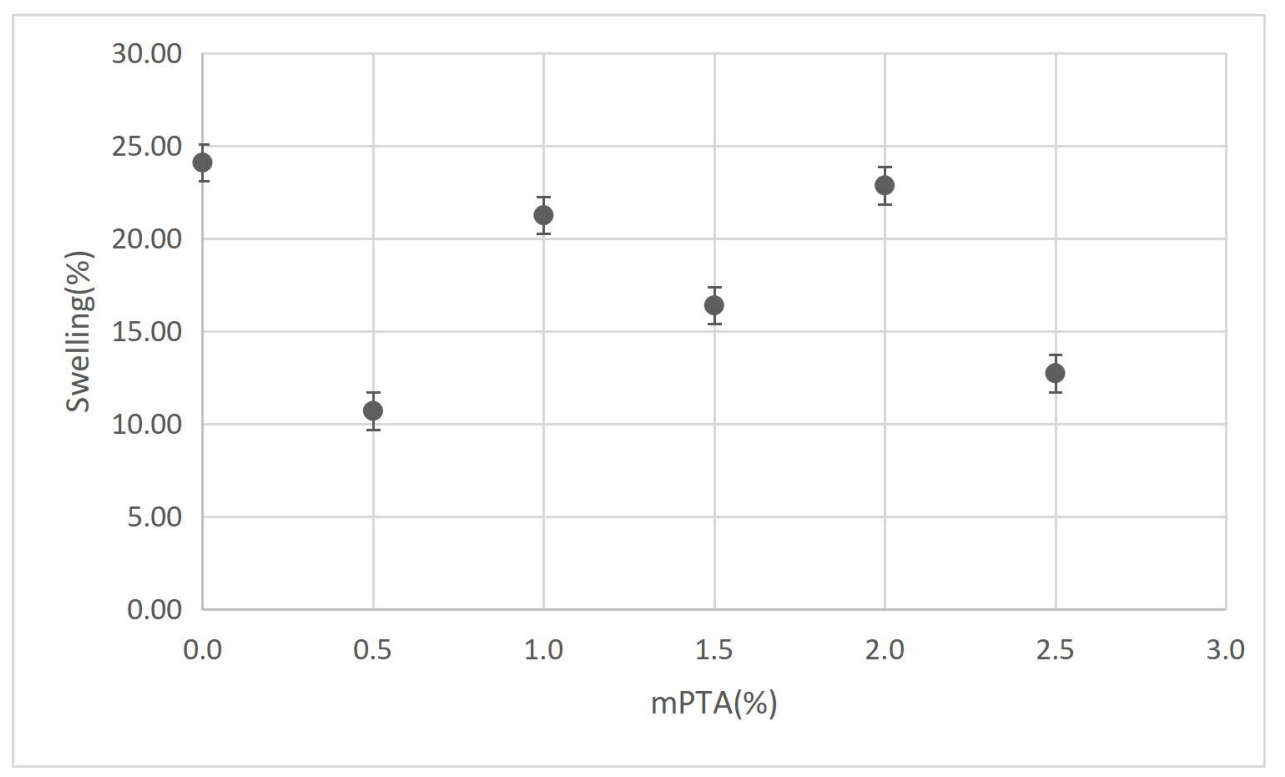

Figure 6 Percentage of swelling of membranes at different mPTA loading 
Bound water is suggested to happen due to the hydrogen bond of $-\mathrm{SO}_{3} \mathrm{H}$ in the SPEEK polymer chain [22]. There are certain factors that can improve SP/mPTA membrane characteristics: (1) good dispersion of mPTA and (2) sufficient water uptake. An increase in the proton conductivity of SP/mPTA nanocomposite membrane can be interpreted in the following way. The absorbed water molecules are present mostly in the ionic cluster domains and ionic cluster channels. In particular, in the ionic cluster channels, the water molecules exist in two different forms.

One is the protonated water (mostly non-freezing bound water) that is bound strongly to the ionic site. The other is free water that occupies the central space free from the influence of the ionic sites. The proton transfer through the ionic cluster channel occurs by two different mechanisms: (1) near the channel wall via the bound water, in which proton is transported by the Grotthuss mechanism, hopping from one ionic site to the other; and (2) via free water by vehicle mechanism, in which proton is facilitated by the water molecules moving through the interconnected central channel space [23]. Grotthuss mechanism plays an important role in this. Therefore, it can be said that the enhanced proton conductivity of the SP/mPTA composite membrane is attributed to the water retention capability.

Figure 5a shows the schematic SPEEK membrane structure without the addition of mPTA fillers. As for the membrane in Figure $5 b$, there is the impact of the addition of mPTA whereby transportation of proton occurs between the sulfonic group of SPEEK matrix and an acid group of mPTA particles when strong hydrogen occurs between them. The Grotthus mechanism (proton hopping) played an important role in bringing the proton from one site to another. The proton was delivered through an ionic cluster channel (free water and $\mathrm{SO}_{3}$ that linked with non-freezing bound water). It is desirable for a membrane to have a high value of bound water (non-freezing bound water) since it is a crucial element in delivering protons. To prove the statement, it can be observed that SP/mPTA showed the highest proton conductivity [23].

\subsection{Proton Conductivity of SPEEK/mPTA Membranes}

Many studies have reported that composite membranes exhibit higher conductivity than their native membranes [24]. Generally, inorganic fillers serve to improve the formation of proton transport channels within the membrane, enhance the water retention capacity, provide additional conductive groups, and consequently improves the conductivity [25]. Figure 7 shows the proton conductivities of the neat SPEEK membrane in comparison with the as-fabricated SPEEK/mPTA membranes. In this case, it clearly shows that the proton conductivity of the composite membrane is reducing with an increase of mPTA loading. This reduction in proton conductivity may be due to the presence of inorganic particles in the membranes [26]. The results show that introducing $\mathrm{mPTA}$ 
particles into the membrane tends to decrease the proton conductivity at a lower temperature [27]. Whereas the water uptake of the modified SPEEK with MPTA was slightly higher than the unmodified SPEEK as shown in Figure 3. The Ionic Exchange Capacity (IEC), water uptake and protonation degree decrease with the increase of mPTA loading and it can be interpreted by the strong action between the sulfonic group and an acid group [28]. When the heteropoly acids were doped in the mPTA, these HPA's can act as the proton carrier and modify the proton conductivity of this membrane. In SPEEK/mPTA composite membranes, there are a number of hydrogen bonds among mPTA and water. The volume of mPTA is so huge that disrupt anions movement as a result of the strength of hydrogen bonds, but protons can transfer along with these hydrogen bonds [29]. With a denser structure and only a little number of protons can make it move through the hydrogen bonds. Compact structure is observed with an increase of mPTA loading as this can be supported by the SEM micrographs obtained as showed in Figure 1. The insertion of inorganic fillers will greatly affect the water uptake of the as-prepared membranes when compared to the pristine membrane. The addition of these inorganic fillers will increase the number of hydrophilic sites such as $\mathrm{OH}-$, COO- and O- [30]. For instance, both $\mathrm{SP} / 1.5 \mathrm{mPTA}$ and $\mathrm{SP} / 2.0 \mathrm{mPTA}$ possessed slightly higher water uptake when compared to pristine SPEEK.
However, the water uptake of SP/2.0mPTA (35.8\%) was higher than SP/1.5mPTA (28.3\%) membranes due to the strong interfacial interaction of covalent cross-linking, which makes SPEEK/mPTA possess a compact network that makes it difficult for water to pass through [31]. The existence of narrower water channels with filler uploading is eventually reducing the proton carrier molecules which has a direct impact on the lower proton conductivity of membranes. SPEEK/mPTA membranes perform at its best when it has high ion exchange capacity (IEC), appropriate water uptake and uniform dispersion of mPTA inorganic fillers [32]. However, when the loading of the MPTA was too high, severe aggregation of the fillers tends to occur. This tends to decrease the effective surface area of the particles, leading to the decrease of the water uptake. Although all of the membranes have small proton conductivity under low relative humidity, these composite membranes (after incorporation of mPTA particles into SPEEK polymer matrix), still show a great increase in fuel cell performance as compared to the pristine SPEEK membrane. The addition of mPTA has also formed a much denser and more closely distribution of mPTA clusters in the SPEEK membrane matrix. It was well understood that the hygroscopic nature of mPTA has successfully contributed to enhancing the singlecell performance by generating a higher power density as display in Figure 8. 


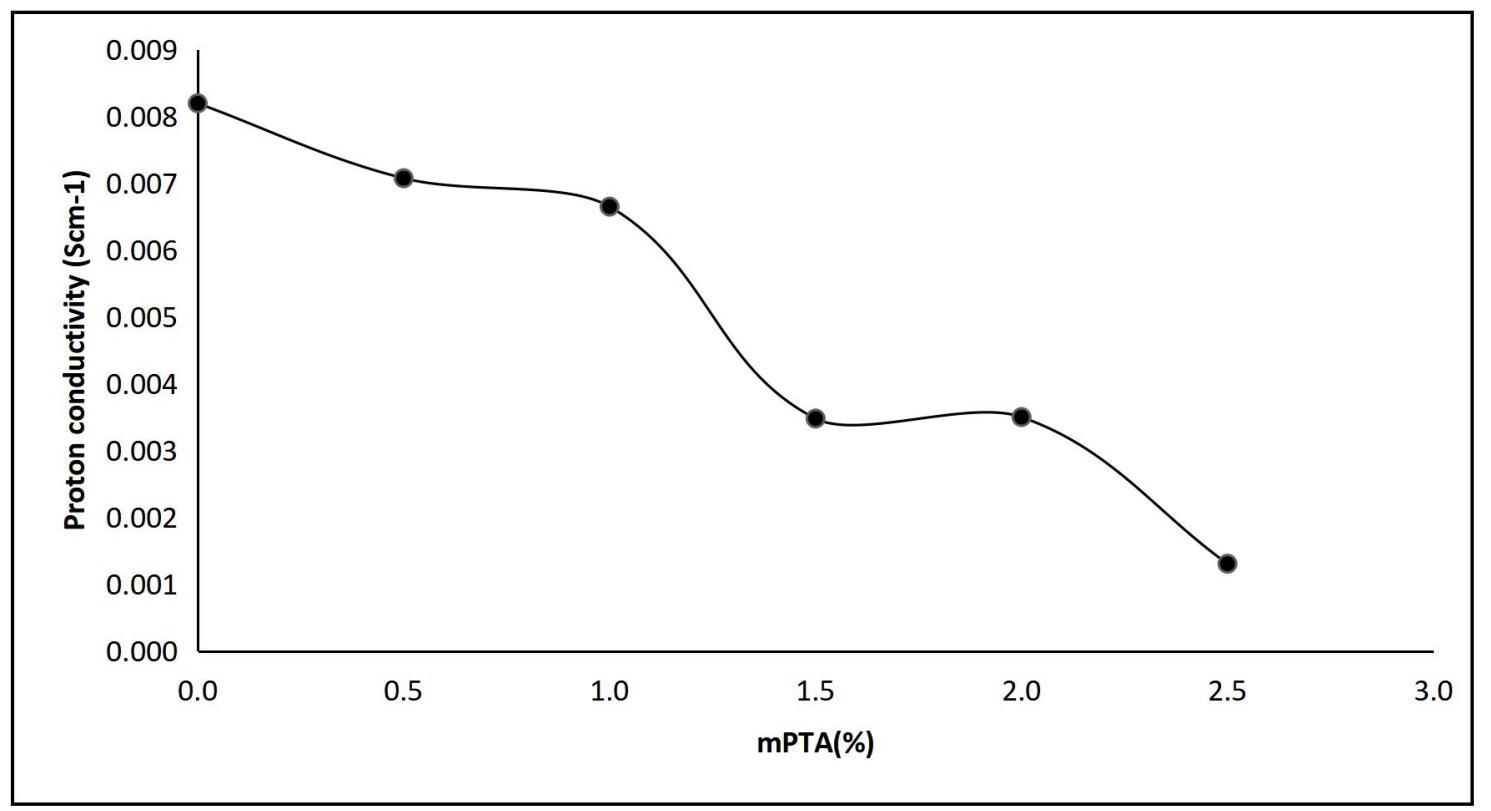

Figure 7 The proton conductivities of neat SPEEK membranes and as-fabricated SPEEK/mPTA membranes

3.5 I-V Polarisation of SPEEK-mPTA Membranes

In order to demonstrate that the SPEEK/mPTA membranes can be employed as PEMs in PEMFCs, the current and power densities were investigated as a function of $\mathrm{mPTA}$ loading. Hypothetically, the fuel cell performance will be at the maximum when proton conductivity is at the highest magnitude. Figure 8 shows the current density-voltage (I- V) and the power density-current density curves for MEAs comprised of SPEEK/mPTA membranes in a single cell operating at $60^{\circ} \mathrm{C}$ and $100 \%$ relative humidity. It can be clearly seen that the SPEEK with 2.0 wt. $\%$ mPTA loading showed the highest open-circuit voltage (OCV) of $0.98 \mathrm{~V}$ as compared to other membranes. This indicates that it has higher membrane durability. This result also revealed that $\mathrm{SP} / 2.0 \mathrm{mPTA}$ has the lowest degradation for the
MEA (membrane electrode assembly) [33]. The PEMFC based on the $\mathrm{SP} / 2.0 \mathrm{mPTA}$ membrane presented the maximum current density of 337.74 $\mathrm{A} / \mathrm{cm}^{2}$ with a maximum power density of $154.4 \mathrm{~mW} / \mathrm{cm}^{2}$. The higher water uptake and proton conductivity of the $\mathrm{SP} / 2.0 \mathrm{mPTA}$ membrane have indeed assisted in the increment of performance of the PEMFCs compared to other membranes. The results also showed a good agreement with the morphological study in which the non-homogeneous dispersion of mPTA at high loading has significantly affected the properties as well as the performance of the resulting membranes. This can be proved based on the $\mathrm{I}-\mathrm{V}$ polarization result that showed SP/2.5mPTA exhibited the lowest power density as compared to other SPEEK $/ \mathrm{mPTA}$ composite membranes. OCV was regarded as a key to evaluate the cathode structure on the PEM durability. Electrode 
structure should be considered as a in addition to the chemical stability of crucial parameter for PEM durability PEMs [33].

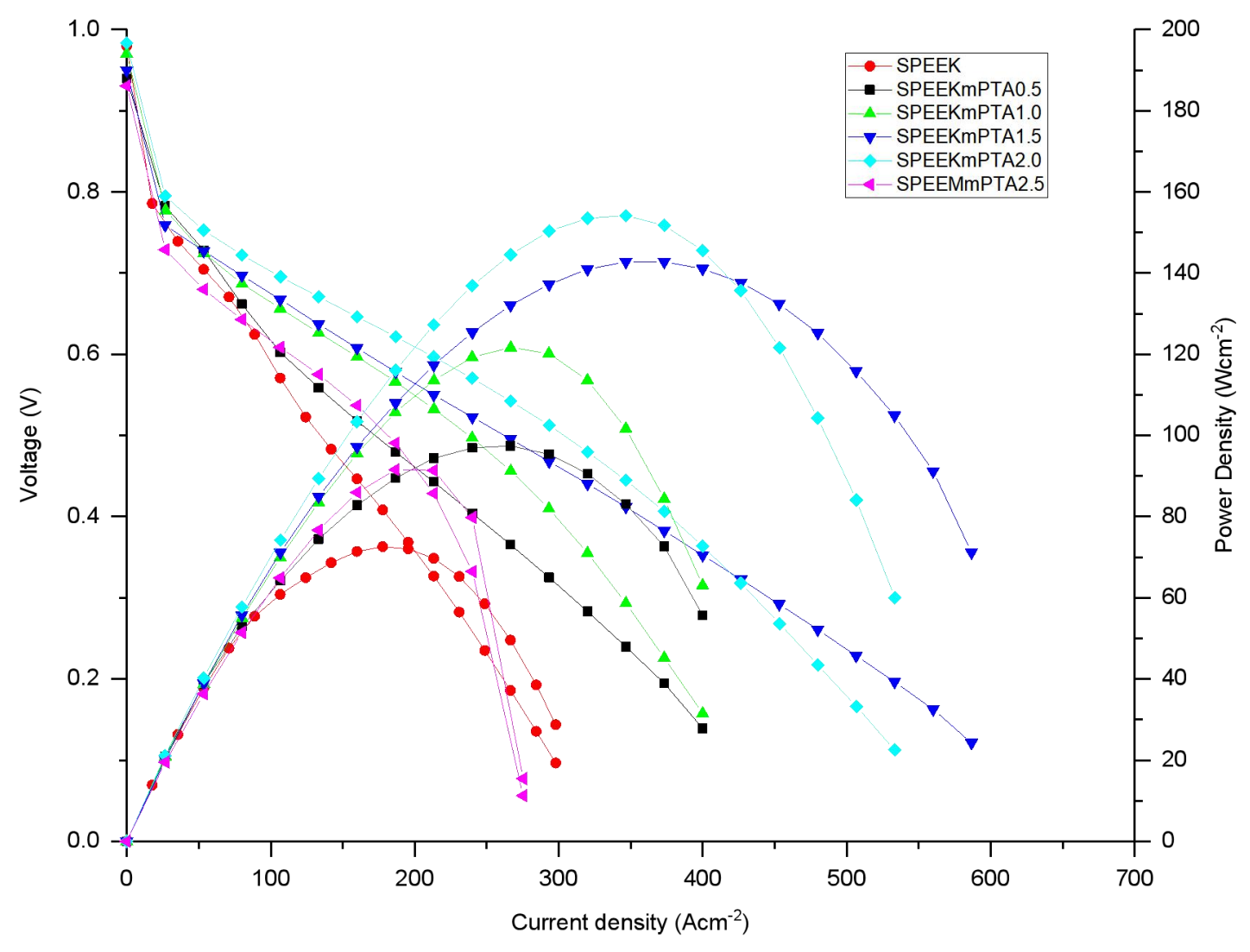

Figure 8 Single cell performance of the SPEEK/mPTA membranes at $60 \mathrm{C}$ under relative humidity of $100 \%$

\subsection{CONCLUSION}

SPEEK/mPTA membranes were successfully fabricated by the solution casting method. The SPEEK/mPTA membranes displayed higher water uptake, lower proton conductivity and better performance than the pristine SPEEK membrane. This was due to the strong hydrogen bonding between the SPEEK and mPTA particles through the interconnection of sulfonic acid and acid functional groups of SPEEK and mPTA, respectively that simultaneously contributed to better effective functional clusters. Particularly, the interconnection of mPTA with ionic regions in the SPEEK matrix and water channels was expected to create continuous pathways for proton transfer thus enhances the proton conductivity of the composite membrane. But, due to the packed structure of the SPEEK/mPTA membranes, the water channels have been reduced which limits the proton transfer. This caused a lower proton conductivity of the as- 
fabricated membranes. The SPEEK/mPTA membrane with a uniform dispersion of mPTA, acceptable water uptake and also with qualified proton conductivity was chosen to perform the single-cell test under PEMFC operation and expected to exhibit a higher performance (current density and power density, respectively) as compared to the neat SPEEK membrane. In conclusion, the incorporation of mPTA at an appropriate amount could increase the overall performance of the membranes and SPEEK/mPTA could be a good candidate for the next fuel cell membrane application.

\section{ACKNOWLEDGMENT}

We are grateful to AMTEC and the School of Chemical and Energy Engineering for the facilities provided that contribute to the success of this study. We also would like to acknowledge the Research Management Center (RMC), UTM for the financial funding through the UTM-TDR research grant (06G88 and 06G87).

\section{REFERENCES}

[1] M. K. Mahapatra and P. Singh. 2014. Chapter 24 - Fuel Cells: Energy Conversion Technology, in Future Energy. 2: 511-547.

[2] M. B. Karimi, F. Mohammadi, and K. Hooshyari. 2019. Recent Approaches to Improve Nafion Performance for Fuel Cell Applications: A Review.
International Journal of Hydrogen Energy. 44(54): 28919-28938.

[3] K. 2019. Amelioration of PEMFC Performance at High Temperature by iIncorporation of Nanofiller (Sepiolite/layered Double Hydroxide) in Nafion Membrane. International Journal of Hydrogen Energy. 44(21): 10666-10676.

[4] A. E. Aslan Gumusdereli, and A. M. Soydan. 2019. Producing of Imidazol Functional $\mathrm{SiO} 2$ Nanoparticles /Nafion Nanocomposite Membranes for PEMFC Applications. Journal of the Faculty of Engineering and Architecture of Gazi University. 34(1): 351-363.

[5] V. Ivanov. 2018. Perspective Non-fluorinated and Partially Fluorinated Polymers for LowTemperature PEM FC, in Proton Exchange Membrane Fuel Cell.

[6] O. Z. Sharaf. and M. F. Orhan. 2014. An Overview of Fuel Cell Technology: Fundamentals and Applications. Renewable and Sustainable Energy Reviews. 32: 810-853.

[7] S. Yoon. 2019. Synthesis of Sulfonation Poly(Npropylsulfonicacid isatin biphenylene) for Polymer Electrolyte Membrane Fuel Cell Containing $\quad \mathrm{SiO} 2$ Nanocomposite Membrane. Journal of Nanoscience and Nanotechnology. 19(3): 15621566. 
[8] A. R. Kim. 2018. Sulfonated Poly Ether Sulfone/Heteropoly Acid Composite Membranes as Electrolytes for the Improved Power Generation of Proton Exchange Membrane Fuel Cells. Composites Part B-Engineering. 155: 272-281.

[9] Y. Rattanachai. 2018. Characterization of of Nafion XL Membrane for PEMFC after VUV Degradation and Titanium Nitride Coating. Nuclear Instruments \& Methods in Physics Research Section B-Beam Interactions with Materials and Atoms. 436: 292-297.

[10] A. A. Abdul Hamid. 2013. Effect of Casting Solvents and Filler Quantity on the Preparation and Physiochemical Properties of PVC - Bentonite Based Composite Polymeric Membranes. Journal Chemical Society of Pakistan.

[11] H. Ilbeygi. 2019. Highly Crystalline Mesoporous Phosphotungstic Acid: A HighPerformance Electrode Material for Energy-Storage Applications. Angew Chem Int Ed Engl. 58(32): 10849-10854.

[12] N. Awang, J. Jaafar, and A. F. Ismail. 2018. Thermal Stability and Water Content Study of Void-Free Electrospun SPEEK/Cloisite Membrane for Direct Methanol Fuel Cell Application. Polymers. 10(2).

[13] H. Ilbeygi. 2019. Highly Crystalline Mesoporous Phosphotungstic Acid: A High-
Performance Electrode Material for Energy-Storage Applications. Angewandte Chemie International Edition. 58(32): 10849-10854.

[14] A. Stassi. 2015. Enhancement of Oxygen Reduction and Mitigation of Ionomer Dry-Out Using Insoluble Heteropoly Acids in Intermediate Temperature PolymerElectrolyte Membrane Fuel Cells. Energies. 8(8): 7805-7817.

[15] M. J. Parnian, S. Rowshanzamir, and J. Alipour Moghaddam. 2018. Investigation of Physicochemical and Electrochemical Properties of Recast Nafion Nanocomposite Membranes Using Different Loading of Zirconia Nanoparticles for Proton Exchange Membrane Fuel Cell Applications. Materials Science for Energy Technologies. 1(2): 146-154.

[16] Y. Yang. 2007. The Influence of Nano-sized TiO2 Fillers on the Morphologies and Properties of PSF UF Membrane. Journal of Membrane Science. 288(1-2): 231-238.

[17] Q. Zhao. 2018. Preparation of High Strength Poly(vinylidene fluoride) Porous Membranes With Cellular Structure Via Vapor-Induced Phase Separation. Journal of Membrane Science. 549: 151164.

[18] M. Y. Chia. 2020. Study on Improvement of the Selectivity of Proton Exchange Membrane via Incorporation of 
Silicotungstic Acid-doped Silica into SPEEK. International Journal of Hydrogen Energy. 45(42): 22315-22323.

[19] P. Salarizadeh. 2016. Influence of Amine-functionalized Iron Titanate as Filler for Improving Conductivity and Electrochemical Properties of SPEEK Nanocomposite Membranes. Chemical Engineering Journal. 299: 320331.

[20] M. J. Parnian, F. Gashoul, and S. Rowshanzamir. 2017. Studies on the SPEEK Membrane with Low Degree of Sulfonation as a Stable Proton Exchange Membrane for Fuel Cell Applications. Iranian Journal of Hydrogen \& Fuel Cell. 3(3): 221-232.

[21] Parisa Salarizadeh, M. J. a. S. P. 2017. Enhancing the Performance of SPEEKpolymer Electrolyte Membrane using Functionalised $\mathrm{TiO} 2$ Nanoparticles with Proton Hoping Sites. Royal Society of Chemistry.

[22] H. Junoh a, J. J., N. A. M. Nor, Nuha Awang, M. N. A. M. Norddin, A. F. Ismail, M. H. D. Othman, Mukhlis and F. A. A. Rahman, N. Yusof, W. N. W. Salleh, R. Naim. 2018. Fabrication of Nanocomposite Membrane via Combined Electrospinning and Casting Technique for Direct Methanol Fuel Cell. Journal of Membrane Science and Research. 4.

[23] N. Awang, J. Jaafar, and A. F. Ismail. 2018. Thermal Stability and Water Content Study of Void-free Electrospun SPEEK/Cloisite Membrane for Direct Methanol Fuel Cell Application. Polymers (Basel). 10(2).

[24] A. Hamid. 2013. Effect of Casting Solvents and Filler Quantity on the Preparation and Physiochemical Properties of PVC - Bentonite Based Composite Polymeric Membranes. Journal Chemical Society of Pakistan. 35.

[25] M. Gnus, G. Dudek, and R. Turczyn. 2018. The Influence of Filler Type on the Separation Properties of Mixed-matrix Membranes. Chem Zvesti. 72(5): 1095-1105.

[26] R. Sigwadi. 2019. The Proton Conductivity and Mechanical Properties of Nafion(R)/ ZrP Nanocomposite Membrane. Heliyon. 5(8): e02240.

[27] R. Sigwadi. 2013. Zirconia Based/Nafion Coposite Membranes for Fuel Cell Applications.

[28] H. Zhang. 2008. Composite Membranes Based on Highly Sulfonated PEEK and PBI: Morphology Characteristics and Performance. Journal of Membrane Science. 308(1): 6674.

[29] L. Li, L. Xu, and Y. Wang. 2003. Novel Proton Conducting Composite Membranes for Direct Methanol Fuel Cell. Materials Letters. 57(8): 14061410.

[30] H. Junoh. 2020. Performance of Polymer Electrolyte Membrane 
for Direct Methanol Fuel Cell Application: Perspective on Morphological Structure. Membranes (Basel). 10(3).

[31] T. Yang. 2018. A Graphene Oxide Polymer Brush Based Cross-linked Nanocomposite Proton Exchange Membrane for Direct Methanol Fuel Cells. RSC Advances. 8(28): 1574015753.
[32] L. Du. 2012. SPEEK Proton Exchange Membranes Modified with Silica Sulfuric Acid Nanoparticles. International Journal of Hydrogen Energy. 37(16): 11853-11861.

[33] B. Choi. 2014. The Effect of Cathode Structures on Nafion Membrane Durability. Journal of The Electrochemical Society. 161(12): F1154-F1162. 